Check for updates

Cite this: RSC Adv., 2018, 8, 25150

\title{
Design and fabrication of vapor-induced superhydrophobic surfaces obtained from polyethylene wax and silica nanoparticles in hierarchical structures
}

\begin{abstract}
Yong Guan, Chenchen Yu, Jiawen Zhu, Rui Yang, Xiang Li, Dafu Wei* and Xiang Xu*
The present work reported a simple and effective approach to fabricate a low-cost, self-cleaning and mechanically durable superhydrophobic coating. The coating was prepared by dip-coating certain substrates in an ethyl acetate suspension of silica nanoparticles $\left(\mathrm{SiO}_{2}\right)$, hydroxyl acrylic resin, crosslinking agent and polyethylene wax (PEW). Through the control of the cooling and drying process, vapor-induced PEW micro-clusters were formed on the surfaces during the evaporation of ethyl acetate, and uniform carpet-like hierarchical structures were finally obtained by properly adjusting the dosage of $\mathrm{PEW}$. Under the synergistic effect of hydrophobic $\mathrm{SiO}_{2}$ nanoparticles and PEW micro-clusters, the composite coating exhibited a remarkable superhydrophobicity with a contact angle of $163^{\circ} \pm 5^{\circ}$ with 25 wt\% content of PEW, as well as preeminent self-cleaning properties against various food liquids. Moreover, the coating still maintained its surface cleanliness when immersed in the cyclohexane or hexadecane, indicating a superior self-cleaning property against solvent-contamination. The mechanical durability test showed that the coating still kept its excellent water repellency after fairly intensive knifescratching, tape peeling and 25 cycles of sandpaper abrasion under $100 \mathrm{~g}$ of loading, indicating a quite admirable mechanical durability. The facile preparation and high-performance of the coating make it quite suitable for manufacture on a large scale, which is favorable for the development of superhydrophobic coatings.
\end{abstract}

Received 25th February 2018

Accepted 1st July 2018

DOI: $10.1039 / c 8 \mathrm{ra01666f}$

rsc.li/rsc-advances

\section{Introduction}

Superhydrophobic technologies derived from nature, typically like the "lotus effect", have attracted much attention around the world both in academic research and in industry applications. Dirt particles on lotus leaves can be easily removed by water droplets due to the hierarchical structures of the surface, which are formed out of a characteristic epidermis and covering of waxes. ${ }^{1}$ Similar non-wettable properties are also prevalent among animals, like water striders and flying insects. ${ }^{2,3}$ Those surfaces show tremendous value in anticorrosion, self-cleaning, drag reduction, oil-water separation and other fields..$^{4-6}$ In general, the superhydrophobic property can be obtained using materials with low surface energy and by constructing morphological structures. ${ }^{7,8}$

Many research works have been reported on artificial superhydrophobic surfaces by controlling the chemical composition of polymers $^{9}$ and metals ${ }^{10,11}$ or by fabricating novel structures using various techniques such as phase separation, ${ }^{12}$ polymer

Key Laboratory for Ultrafine Materials of Ministry of Education, School of Materials Science and Engineering, East China University of Science and Technology, Shanghai 200237, P. R. China. E-mail:dfwei@ecust.edu.cn; xiangxu@ecust.edu.cn imprinting, ${ }^{13}$ sol-gel processing, ${ }^{14}$ chemical etching ${ }^{15}$ and others. ${ }^{16}$ However, these methods are usually complex and timeconsuming. Therefore, it is crucial to develop a simple and effective approach to fabricate a superhydrophobic coating which is suitable for the manufacture in a large scale. Dip-coating is thought to be one of the most promising methods since it is lowcost and less demanding for production facilities. Furthermore, considering that the components applied in superhydrophobic surfaces like perfluoroalkylsilane are usually expensive and ecologically unfriendly, ${ }^{17-20}$ it is more provident to develop a superhydrophobic surface using fluorine-free and sustainable materials. $^{21-24}$

Fabricating hierarchical structures in micro- and nanoscale is an important concept to create a superhydrophobic coating. Inorganic nanoparticles like $\mathrm{SiO}_{2}$ and $\mathrm{TiO}_{2}$, combined with organic polymers, ${ }^{25}$ are usually used to improve the hydrophobicity and surface roughness of the coating. Nevertheless, those hierarchical structures are usually fragile which exhibited poor resistance to mechanical scratch and abrasion since nanoparticles are difficult to cooperate with the organic polymer materials.

One effective solution is to acquire a strong bonding of inorganic nanoparticles to the matrix resin by adding a cross- 
linking agent, ${ }^{26,27}$ endowing the coatings with superhydrophobicity and abrasion resistance. Another useful method is to apply an external process like thermal annealing, to form "welding" between polymer matrix and particle interfaces, ${ }^{28,29}$ which could greatly enhance the durability of hierarchical structures.

Inspired by the epicuticular wax on lotus surface, some efforts have been made to render wax with superhydrophobicity through the fabrication of hierarchical structures, among which a typical method is phase separation. Some researchers used a combination of solvent and nonsolvent to create phase separation of wax. Lu et al. ${ }^{30}$ prepared a low-density polyethylene superhydrophobic surface by controlling its crystallization behavior in a xylene-cyclohexanone mixture. However, those solvents were usually toxic and harmful, thus limiting the application of coatings. Other researchers chose mixed wax for the phase separation during drying process. Zhang et al. ${ }^{31}$ endowed paper surface with superior superhydrophobicity via the phase separation of green-based waxes. Wang et $a l^{32}$ used the beeswax and carnauba wax to fabricate nontoxic superhydrophobic coatings that could be applied on food containers. Though hierarchical bulbiform shapes in microscale could be generated in this way, the components were stacked on the surface randomly thus might be easily buried underneath, which would lead to structure defects.

In this work, a vapor-induced method using fluorine-free materials to fabricate superhydrophobic surfaces was reported. Organic components (hydroxyl acrylic resin, crosslinking agent as well as PEW) and inorganic components $\left(\mathrm{SiO}_{2}\right)$ were mixed in ethyl acetate to make a homogeneous suspension for dip-coating. The coating was endowed with a micro/nano-structure and enhanced roughness simultaneously through a simple control of preparation process, boosting the operation flexibility compared with the complicated methods reported previously. PEW could be dispersed evenly in ethyl acetate with other components due to its low melting point, therefore eliminating the usage of toxic organic solvent. By the virtue of the high reactivity of isocyanate groups with silanol and hydroxyl groups, hydroxyl acrylic resin and $\mathrm{SiO}_{2}$ were cured by the cross-linking agent as a basic hydrophobic rough layer onto the surface of glass slides. Therefore, a rather reinforced coating was formed, partly offsetting the mechanical weakness of hydrophobic coatings. Moreover, PEW emulsions were crystallized into uniform whiskers under the induction of ethyl acetate vapor, and a carpet-like surface was formed with the increase of PEW content, which greatly increased the surface roughness and endowed the coating with superhydrophobicity and antifouling ability. Additionally, different preparation conditions were compared to explore their effects on surface morphology and superhydrophobicity. The coatings were characterized by scanning electron microscope, FT-IR and contact angle/interface system. Self-cleaning test and mechanical durability test were used to evaluate the performance of the composite coating.

\section{Experimental}

\section{Materials}

Polyethylene wax (PEW-G500) was supplied by Kaikai Technology \& Trade Co., Ltd. (China). Hydroxyl acrylic resin (AMS 2788) was purchased from Guangzhou Yuemei Chemical Co., Ltd. (China). Silica nanoparticles $\left(\mathrm{SiO}_{2}-\mathrm{R} 812\right)$ with an average particle size (7-40 nm) was provided by Evonik Industries AG (Germany). Hexamethylene diisocyanate trimer (HDI trimerN3390) acting as a cross-linking agent was bought from Bayer (Germany), and ethyl acetate in analytical purity was provided by Shanghai Titan Scientific Co., Ltd. (China). All chemicals were used without further purification.

\section{Preparation of SPEW coatings}

$3 \mathrm{~g}$ of $\mathrm{SiO}_{2}$, and $1.2 \mathrm{~g}$ of hydroxyl acrylic resin were ultrasonically dispersed in $100 \mathrm{ml}$ ethyl acetate for $30 \mathrm{~min}$ to form a homogeneous suspension. A series amount of PEW (10 wt\% to $25 \mathrm{wt} \%$ ) were added into the suspension and the mixture was magnetically stirred in a water bath at $70{ }^{\circ} \mathrm{C}$ for $30 \mathrm{~min}$, then $0.4 \mathrm{~g}$ of HDI trimer was added into the suspension followed by another $5 \mathrm{~min}$ stirring. After that, the mixture was cooled to $30{ }^{\circ} \mathrm{C}$ at room temperature, and the hybrid coating was fabricated by a simple dip-coating method using glass slides, which were washed with ethyl acetate successively for several times. Then the coated slides were put in the oven at $80{ }^{\circ} \mathrm{C}$ for $2 \mathrm{~min}$, and finally cured at room temperature for $24 \mathrm{~h}$ to obtain a silicaPEW (SPEW) composite coating.

\section{Characterization of SPEW coatings}

Surface morphology. The surface morphology was observed by a scanning electron microscope (SEM, S-3400N, Hitachi) with an acceleration voltage of $15 \mathrm{kV}$ after gold-sputtering for $1 \mathrm{~min}$. Coverslips were used to prepare SPEW coating samples for goldsputtering.

Structure. The spectra of samples were recorded on a FT-IR analyzer (Nicolet 6700, Thermo Electron) from 4000 to $500 \mathrm{~cm}^{-1}$. $\mathrm{SiO}_{2}$ and hydroxyl acrylic resin were ground with $\mathrm{KBr}$ respectively to form a pellet to record the FT-IR spectra. The reaction products of $\mathrm{SiO}_{2}$ and hydroxyl acrylic resin with HDI trimer were reserved on a coverslip and recorded in a reflection mode. HDI trimer was coated on a $\mathrm{KBr}$ crystal window to obtain the FT-IR spectra in a coating method.

Contact angle and sliding angle. Contact angle and sliding angle measurements were performed using a contact angle/ interface system (JC2000D3, Shanghai Zhongchen Digital Technical Apparatus) at room temperature. A 5-8 $\mu$ l of water droplet was placed onto the samples and the final value was obtained from the average of 5 measurements in different locations for each sample.

Self-cleaning test. Various food liquids including ketchup, orange juice, cola, vinegar and yogurt were used for the repelling test. The liquid droplets were dropped from top of coated slides, which were put in a certain gradient to observe the repelling performance along the rolling paths. For dirt-removal test, $3 \mathrm{~g}$ of dusts in diameter of $c a .500 \mu \mathrm{m}$ were put onto the 
samples to create an artificially contaminated surface. Then the samples were immersed in cyclohexane or hexadecane, and water dyed by ink were dropped onto the coated surface to test the self-cleaning property.

Mechanical durability test. Knife-scratch, sandpaper abrasion and tape peeling tests were carried out to test the mechanical durability of the SPEW coating. For knife-scratch test, sparse and intensive cross-scratches were applied respectively on the surfaces to evaluate the scratch resistance property. For the sandpaper abrasion resistance test, the coated glass slide $(25 \mathrm{~mm} \times 75 \mathrm{~mm})$ under $100 \mathrm{~g}$ of loading was placed facedown to the 2000 mesh sandpaper. The surface was moved back and forth along the ruler from one side to another parallel to the sandpaper. $10 \mathrm{~cm}$ of the moving distance was recorded as one cycle of the abrasion test. In the tape peeling test, an adhesive tape (Scotch-600 tape) was glued to the sample surface and tightly pressed, then was removed by peeling. The resulting surface was observed with SEM. Water was dropped on different points to measure the contact angles after knife-scratch, sandpaper abrasion and tape peeling tests.

\section{Results and discussion}

\section{Molecular structures of nanoscale coatings}

The FTIR spectra of the components $\mathrm{SiO}_{2}$, hydroxyl acrylic resin, HDI trimer as well as the reaction products are shown in Fig. 1. Based on the relative absorbance intensity of functional groups, it was discovered that hydroxyl groups of acrylic resin $3480 \mathrm{~cm}^{-1}$ and silanol groups of $\mathrm{SiO}_{2}$ at $3414 \mathrm{~cm}^{-1}$ almost disappeared due to the possible reaction with isocyanate groups in HDI trimer, while $\mathrm{C}-\mathrm{H}$ group at $2935 \mathrm{~cm}^{-1}$ in the acrylic resin and $\mathrm{Si}-\mathrm{O}-\mathrm{Si}$ groups at $1086 \mathrm{~cm}^{-1}$ in $\mathrm{SiO}_{2}$ still remained. In the FTIR spectrum of HDI trimer, two intense peaks at $1688 \mathrm{~cm}^{-1}$ and $2277 \mathrm{~cm}^{-1}$ were assigned to stretching vibrations of $\mathrm{C}=\mathrm{O}$ bond and -NCO groups, respectively. However, the absorbance intensity ratio of $-\mathrm{NCO}$ groups to $\mathrm{C}=\mathrm{O}$ bond dramatically

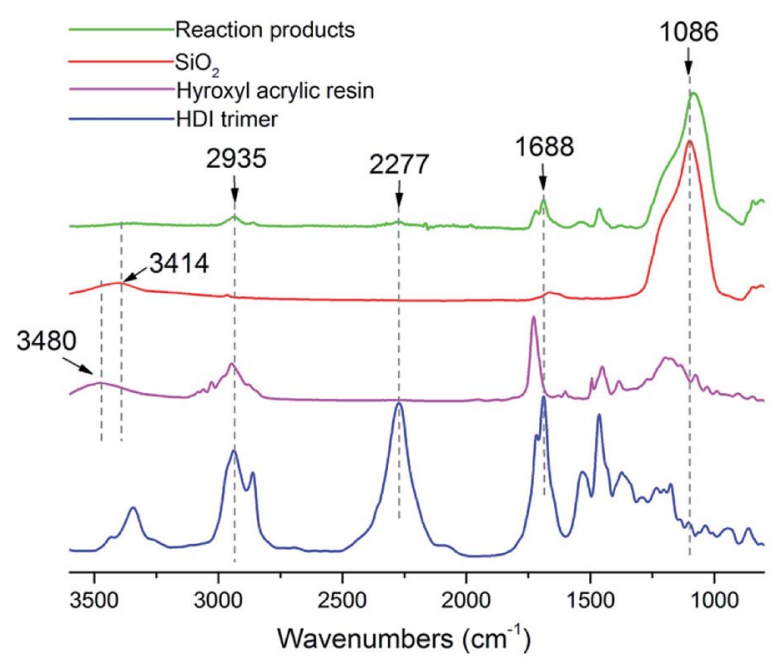

Fig. 1 FTIR spectra of $\mathrm{HDI}$ trimer, hydroxyl acrylic resin, $\mathrm{SiO}_{2}$ and reaction products.

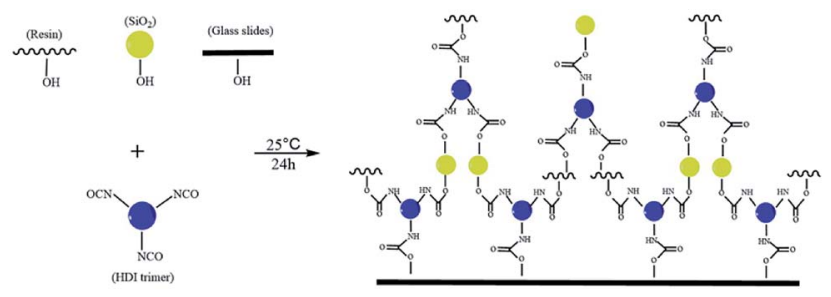

Scheme 1 Possible bonding structures among $\mathrm{SiO}_{2}$, hydroxyl acrylic resin and $\mathrm{HDI}$ trimer on glass slides.

decreased in reaction products compared with that of HDI trimer, which indicated that most of the -NCO groups were consumed during the curing process and transferred into a -O$\mathrm{C}(\mathrm{O})-\mathrm{NH}$ - structure. Based on the above analysis, the possible chemical bondings among $\mathrm{SiO}_{2}$, hydroxyl acrylic resin, HDI trimer and glass slides are displayed Scheme 1. The newly formed $-\mathrm{O}-\mathrm{C}(\mathrm{O})-\mathrm{NH}$ between glass slides and HDI trimer helped to form a stable and robust composite coating.

\section{Micro-morphology of superhydrophobic SPEW composite coatings}

The SEM images as well as the images of water contact angles of surface of SPEW coatings with varied PEW weight ratio are shown in Fig. 2. The particular PEW microscale structures were observed with the addition of PEW. When $10 \mathrm{wt} \%$ of PEW was added into the mixture, a small amount of PEW whiskers randomly dispersed on the coating surface, displaying a contact angle of $131^{\circ} \pm 2^{\circ}$ (Fig. 2a $\mathrm{a}_{1}$ ). With the increase of PEW content from $15 \mathrm{wt} \%$ to $20 \mathrm{wt} \%$, more PEW clusters formed and the surfaces became much rougher (Fig. $2 \mathrm{~b}_{1}$ and $\mathrm{c}_{1}$ ), showing contact angles rising from $147^{\circ} \pm 3^{\circ}$ to $153^{\circ} \pm 1^{\circ}$. The hydrophobic coating was successfully transferred into a superhydrophobic coating. As the PEW content was continuously increased to $25 \mathrm{wt} \%$, a homogeneous micro- and nanoscale hierarchical structure was fabricated (Fig. $2 \mathrm{~d}_{1}$ ), and the contact angle further increased to $163^{\circ} \pm 5^{\circ}$. In the SEM expansion images of coatings (Fig. $2 \mathrm{a}_{2}-\mathrm{d}_{2}$ ), the PEW whiskers in Fig. $2 \mathrm{~d}_{2}$ was observed to provide a more efficient air gap compared with those of other coatings, and acted as a full-scale protection to prevent the coating from being contaminated.

\section{Formation mechanism of superhydrophobic surface}

The formation mechanism of surface morphology is illustrated in Scheme 2. The mixture was stirred at $70{ }^{\circ} \mathrm{C}$ until a uniform suspension was formed. The inorganic hydrophobic nanoparticles were easily suspended in the PEW emulsion due to similar polarities. In the followed cooling process until $30{ }^{\circ} \mathrm{C}$, PEW crystal nucleuses in microscale were gradually deposited out of the mixture. Then glass slides were fastly dip-coated and put immediately in an oven at $80{ }^{\circ} \mathrm{C}$ for $2 \mathrm{~min}$. As the solvent evaporated during the drying process, $\mathrm{SiO}_{2}$ particles slowly precipitated onto the interface between mixture and slide due to a higher density, and formed a loose hydrophobic layer. Meanwhile, the solvent underneath was evaporated in a slower 

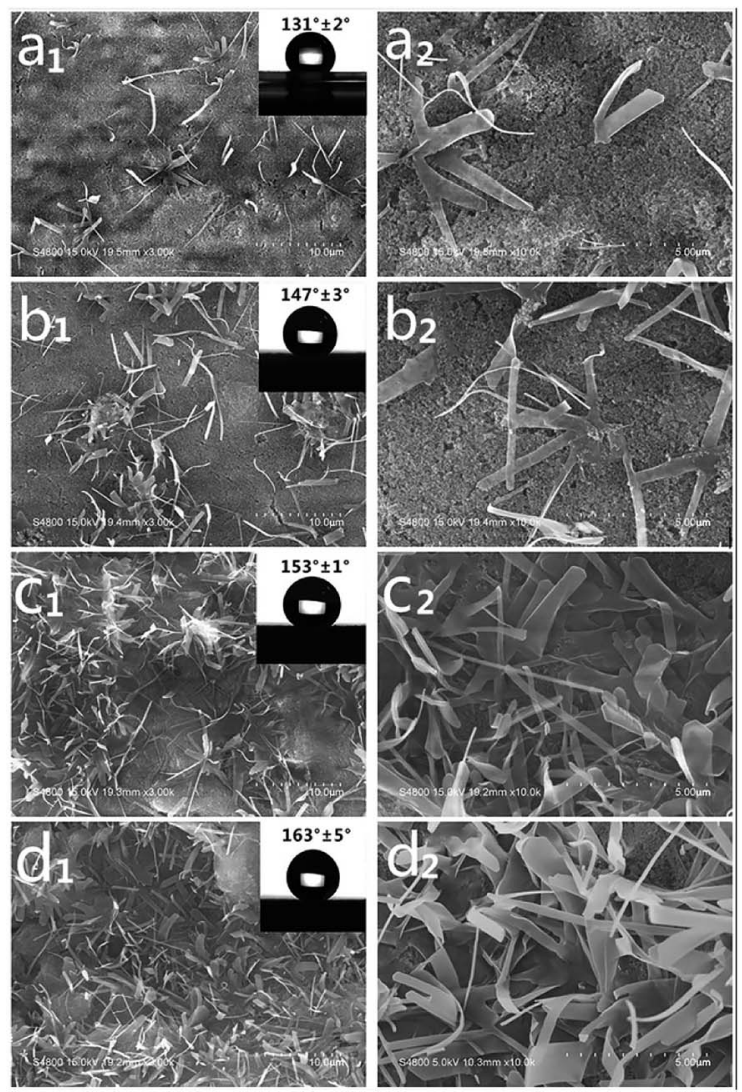

Fig. 2 SEM images of micro-nanoscale structures of SPEW coatings with various PEW dosages. $\left(a_{1}\right) 10$ wt\%, $\left(b_{1}\right) 15$ wt\%, $\left(c_{1}\right) 20$ wt\%, $\left(d_{1}\right)$ 25 wt\%; $\left(a_{2}-d_{2}\right)$ expansion images from $\left(a_{1}-d_{1}\right)$.
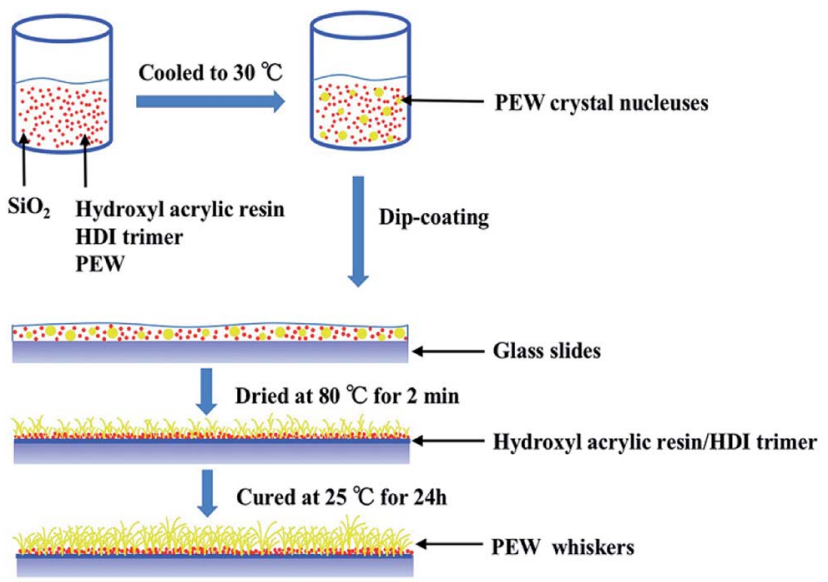

Scheme 2 Schematic illustration for the formation of superhydrophobic surface.

rate through the voids among nanoparticles. It is worth mentioning that by the virtue of a lower density, PEW crystal nucleuses gradually migrated to the upper layer during the evaporation of solvent. Since the drying temperature was 10$20{ }^{\circ} \mathrm{C}$ higher than the melting point of PEW, crystal nucleuses were transferred into a near molten state, and then slowly shaped to small whiskers induced by ethyl acetate vapor. The samples were then slowly cured at room temperature for $24 \mathrm{~h}$, which provided a condition for the growth of PEW whiskers. Finally, a micro-nanoscale composite coating with relatively high surface roughness was fabricated.

In order to further investigate the influence of preparation conditions on surface morphologies, different coatings with $25 \mathrm{wt} \%$ of PEW were prepared, as the results are shown in Table 1 and Fig. 3. Sample E was the same as sample d in Fig. 2. Compared with sample C, D and E to cool to $30^{\circ} \mathrm{C}$, it took more time for sample A to cool to $25^{\circ} \mathrm{C}$, which resulted in lager crystal nucleuses of PEW. These crystals might be difficult to be transferred into a near molten state at $80{ }^{\circ} \mathrm{C}$ in $2 \mathrm{~min}$, affecting the re-shaped process induced by ethyl acetate vapor, thus depositing in a random shape with a diameter of 1-3 $\mu \mathrm{m}$ on the surface, as shown in Fig. 3a.

In contrast with sample A, many small PEW whiskers in a diameter of 100-300 $\mathrm{nm}$ formed on the surface of sample C (Fig. 3c). It might be owed to the shearing effect from stirring during the cooling process, which prompted the formation of many small crystal nucleuses. It was noticed that the coating of sample B, which was dried directly without cooling process, was netlike and consisted of entangled pores with a diameter in the range of several micrometers (Fig. 3b). It confirmed that PEW suspension under a molten state was thermodynamically unstable when evaporating the solvent. As a result, phase separation would occur to form a PEW rich phase and a PEW poor phase. The rich phase solidified and formed the matrix after drying the solvent, whereas the PEW poor phase formed the pores. In addition, the hierarchical structures fabricated by present method remarkably increased the surface roughness, displaying an increased water contact angle to $141^{\circ} \pm 1^{\circ}$. Since the drying temperature was much higher than the melting point of PEW, a similar effect on the surface morphologies was observed by raising the drying temperature or prolonging the drying time. Sample D was dried for a longer time compared with other coatings. As a result, PEW crystals were remelted into liquid drops during the evaporation of solvent. However, those drops wrapped with nanoparticles could not aggregate together and then were stacked randomly on the surface, as shown in Fig. 3d. Therefore, the detailed surface morphologies could be controlled by changing the cooling and drying conditions during the process, which was also reported by other researches, such as netlike hollow structures resulting from phase separation. ${ }^{33}$ Nevertheless, the carpet-like morphology was seldom illustrated, and its performance was rarely investigated.

\section{Applications of SPEW coatings}

The coating of $25 \mathrm{wt} \%$ PEW content was chosen to investigate its applications due to its more compact micro structures and superior superhydrophobicity. Various substrates were used for the characterization of superhydrophobicity, including filter paper, cotton, textile, glass slides and tinplate, as shown in Fig. 4. Dyed water droplets were placed on the white coated surfaces and the uncoated surfaces, respectively. The composite 
Table 1 Process parameters of different coatings

\begin{tabular}{|c|c|c|c|c|}
\hline Samples & Process & Cooling & Drying & Contact angles \\
\hline A & Cooling/drying & To $25^{\circ} \mathrm{C}$, no stirring & $80^{\circ} \mathrm{C}, 2 \mathrm{~min}$ & $132^{\circ} \pm 2^{\circ}$ \\
\hline B & Drying & - & $80^{\circ} \mathrm{C}, 2 \mathrm{~min}$ & $141^{\circ} \pm 1^{\circ}$ \\
\hline $\mathrm{D}$ & Cooling/drying & To $30^{\circ} \mathrm{C}$, no stirring & $80^{\circ} \mathrm{C}, 5 \mathrm{~min}$ & $135^{\circ} \pm 2^{\circ}$ \\
\hline $\mathrm{E}$ & Cooling/drying & To $30^{\circ} \mathrm{C}$, no stirring & $80^{\circ} \mathrm{C}, 2 \mathrm{~min}$ & $163^{\circ} \pm 5^{\circ}$ \\
\hline
\end{tabular}
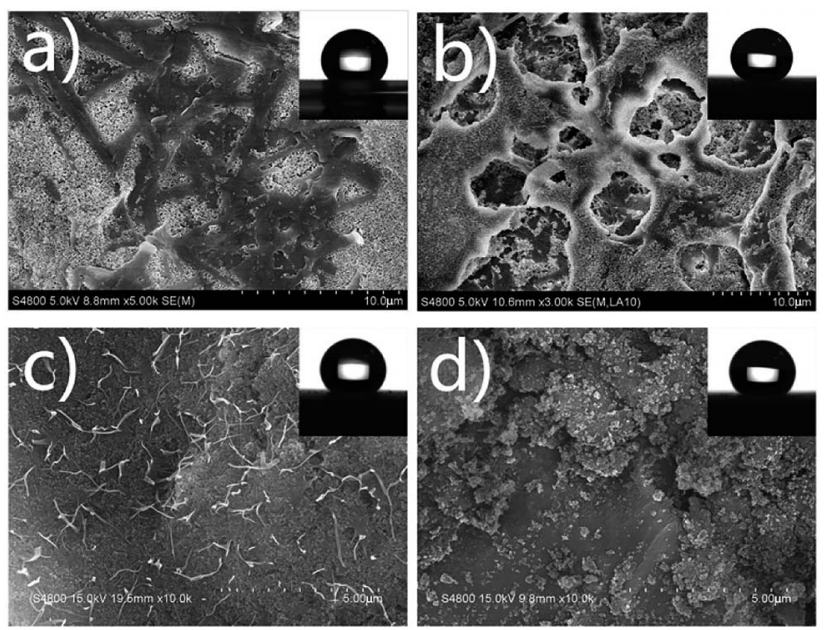

Fig. 3 SEM images of coatings prepared in different conditions. (a) Cooled to $25^{\circ} \mathrm{C}$, (b) without cooling process, (c) cooling with stirring, (d) dried at $80^{\circ} \mathrm{C}$ for $5 \mathrm{~min}$

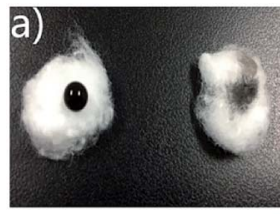

d)

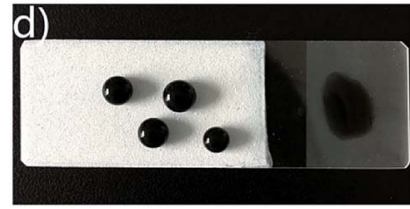

Fig. 4 Dyed water droplets on the coated and uncoated surfaces of cotton (a), filter paper (b), textile (c), glass slide (d) and tinplate (e).

coating on various substrates exhibited an outstanding superrepellency property to water droplets, which still maintained their sphere-like shapes on different points of coated surfaces. For some substrates with certain inherent interspace, such as cotton and textile (Fig. 4a and c), water droplets easily penetrated through those uncoated surfaces. However, on all the coated surface droplets maintained their sphere-like shapes and easily rolled off from the surfaces, indicating a perfect adaptability of the composite coating on both hard (glass and tinplate) and soft (filter, paper, cotton and textile) substrates. It should be noted that the coated surfaces were white and nontransparent, which may partly limit their practical applications.

\section{Self-cleaning property of SPEW coatings}

In order to further evaluate the performance of composite coating, various liquids were used for the repelling test, such as some food liquids in the reported work. ${ }^{34}$ Fig. 5 showed the pictures of these liquids on the composite coating, as well as the corresponding contact angles and sliding angles. Ketchup, orange juice, cola, vinegar and yogurt maintained the spherical shapes with high contact angles and low sliding angles while olive oil and cooking oil spread out on the surface, showing a lipophilic property of the coating. Since PEW and hydrophobic $\mathrm{SiO}_{2}$ are both lipophilic, it's reasonable that the coating could not repel oil. And further addition of materials with low surface energy could be one of the potential methods to vest the coating with oleophobic property. ${ }^{35,36}$

Rolling processes of various liquids are shown in Fig. 6, with contact angle images placed on the bottom right corner. Coated surfaces were put in a certain slope before test. For ketchup and yogurt tests, the coated slides were put in a higher slope $\left(45^{\circ}\right.$ and $30^{\circ}$ ) for a smoother rolling process. When those liquids were dropped on the coating, they could completely roll off from the surfaces with no residual trace. Therefore, the coating could maintain its surface cleanness against both light liquids and thick liquids.

Self-cleaning tests under hydrophobic solvent system was rarely reported probably due to the loss of water repellency when superhydrophobic surfaces are contaminated by hydrophobic solvent. ${ }^{37}$ In this work, the uncoated and coated glass slides with dust contamination were immersed in cyclohexane and the processes of removing the dust were recorded when water droplets rolled over the dust to further evaluate the selfcleaning property of the coating, as presented graphically in Fig. 7. Fig. $7 a_{1}-a_{3}$ showed that when water droplets rolled off from glass slides, they spread in a random shape, and the dust
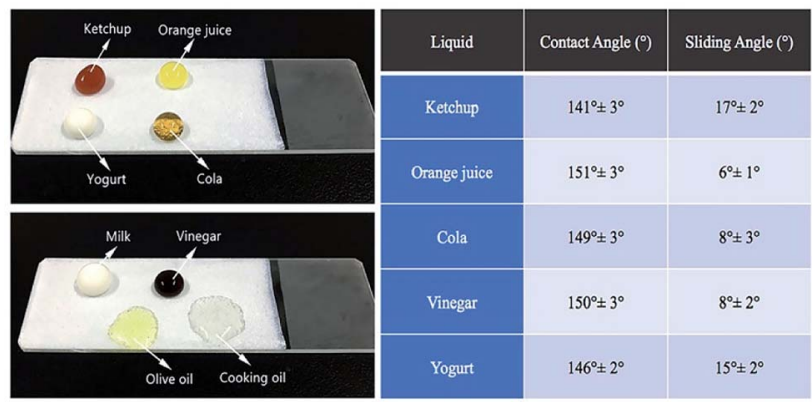

Fig. 5 Various liquid repelling tests on the coated surfaces (left) and their contact angles/sliding angles (right). 

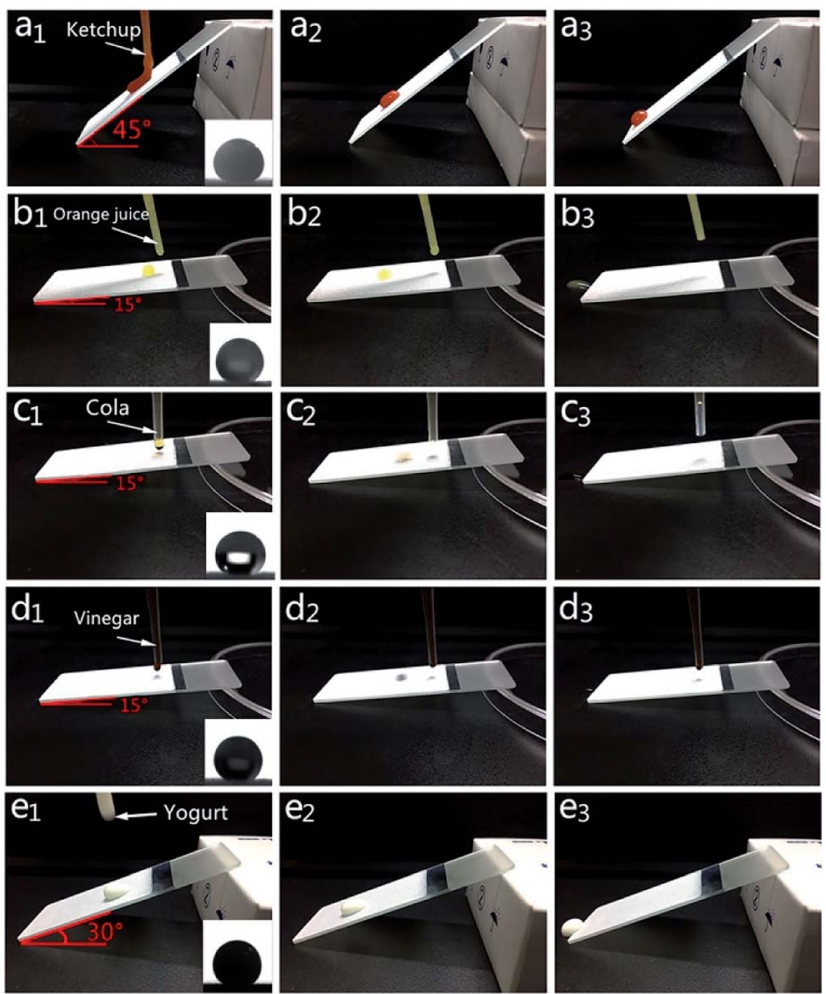

Fig. 6 Rolling processes of ketchup $\left(a_{1}-a_{3}\right)$, orange juice $\left(b_{1}-b_{3}\right)$, cola $\left(c_{1}-c_{3}\right)$, vinegar $\left(d_{1}-d_{3}\right)$, yogurt $\left(e_{1}-e_{3}\right)$ on the coated surfaces.

still remained along the path. In contrast, the composite superhydrophobic coating allowed water droplets to take the dust away from its rolling and bouncing path, as shown in Fig. $7 b_{1}-b_{3}$, indicating that the self-cleaning property was still retained even after cyclohexane-contamination. Changing cyclohexane to hexadecane, the same results of dust successfully removed on the coating surface were observed, further indicating an excellent self-cleaning property of the SPEW coating.

\section{Durability of SPEW coatings}

Durability of superhydrophobic coatings is a crucial factor that limits their widespread applications, since micro- and nanostructures may be destroyed when faced with mechanical
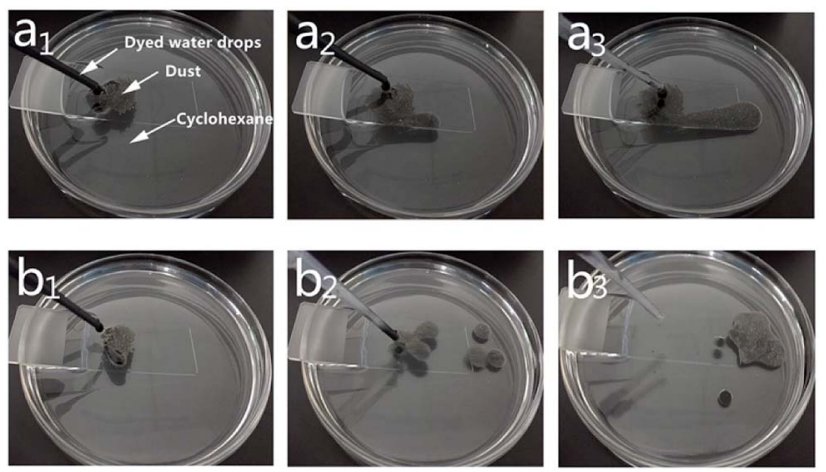

Fig. 7 Self-cleaning test on uncoated surface $\left(a_{1}-a_{3}\right)$ and coated surface $\left(b_{1}-b_{3}\right)$ when surfaces immersed in cyclohexane.

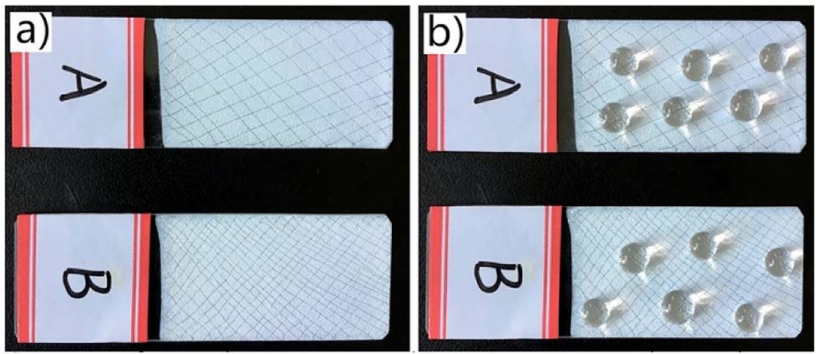

Fig. 8 Knife-scratch test. Sparse and intensive cross-scratches on the coated surfaces (a), water droplets on the scratched surfaces (b).

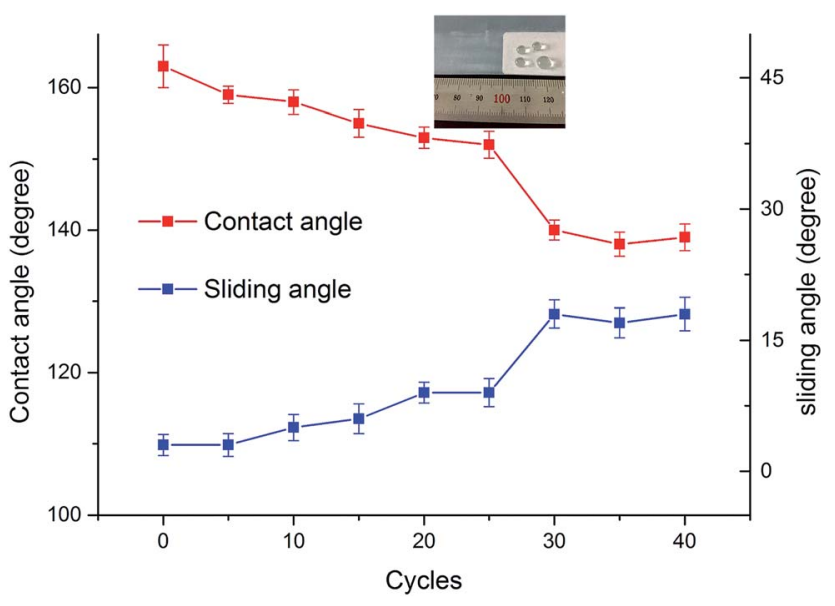

Fig. 9 Water contact angles and sliding angles of SPEW coating during 40 abrasion cycles.

friction, leading to the loss of superhydrophobicity. Here, knifescratch, sandpaper abrasion and tape peeling tests were used to evaluate the mechanical durability of the SPEW coating. As shown in Fig. 8a, sparse (A) and intensive (B) cross-scratches were applied respectively on the coated surfaces to imitate the scratch conditions in practical applications, and water droplets were placed on the surfaces after knife-scratch (Fig. 8b). It was noticed that the coating still retained their non-wetting property, regardless of sparse or intensive cross-scratches. The contact angles were $158^{\circ} \pm 2^{\circ}$ (A) and $153^{\circ} \pm 2^{\circ}$ (B) respectively, indicating that scratching did not induce a significant decrease of the superhydrophobicity on the SPEW coating.

In sandpaper abrasion test, water contact angles and sliding angles were recorded after a certain number of abrasion cycles, shown in Fig. 9, and SEM images before and after 25, 40 abrasion cycles are shown in Fig. 10. When the PEW whiskers that protruded onto the SPEW surface (Fig. 10a) were first removed, the contact angles decreased a little from $163^{\circ} \pm$ $5^{\circ}$ to $159^{\circ} \pm 1^{\circ}$. As the number of abrasion cycles increased, part of PEW micro structures was destroyed, resulting in the decrease of contact angles and increase of sliding angles. However, the coating still kept its superhydrophobicity after 25 cycles of abrasion, with a contact angle of $152^{\circ} \pm 3^{\circ}$ and sliding angle of $9^{\circ} \pm 2^{\circ}$. Fig. 10b showed that some PEW whiskers still remained and $\mathrm{SiO}_{2}$ nanoparticles stacked on the whiskers, which helped to maintain the superhydrophobicity of coating. 

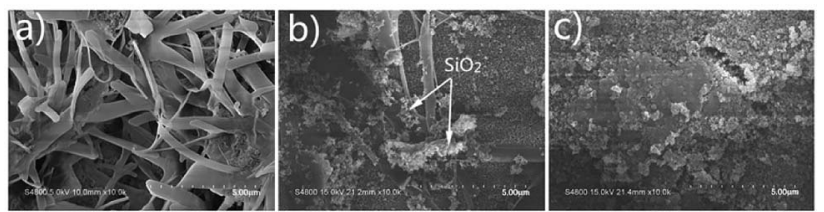

Fig. 10 SEM images of coatings before (a) and after 25 abrasion cycles (b), 40 abrasion cycles (c)
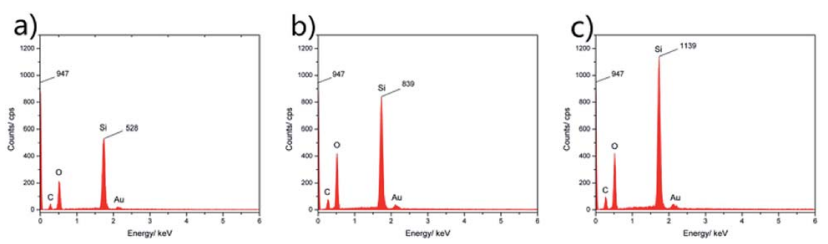

Fig. 11 EDX spectra of coatings before (a) and after 25 abrasion cycles (b), 40 abrasion cycles (c)

However, a significant decrease of contact angle was noticed after 30 cycles of abrasion, leading to the loss of superhydrophobicity. It might owe to the destruction of PEW whiskers microscale structures after multiple abrasion cycles, leaving the nanoscale structures consisting of $\mathrm{SiO}_{2}$ to resist the abrasion from sandpaper, as SEM image shown in Fig. 10c. In the test after 30 cycles, the contact angles and sliding angles had no remarkable change, showing the increasing durability of nanoscale layer, which might relate with the firm bonding of $\mathrm{SiO}_{2}$ with hydroxyl acrylic resin due to the reaction among $\mathrm{SiO}_{2}$, hydroxyl acrylic resin and HDI trimer.

EDX spectra of the coatings before and after 25, 40 abrasion cycles were also recorded to study the changes of surface element content, shown in Fig. 11. For the surface almost covered with PEW whiskers before abrasion, the atomic signal of Si was relatively weak. Compared with the benchmark peak at $0 \mathrm{keV}$, the samples after 25 and 40 abrasion cycles showed much higher Si content on their surfaces, due to a raising number of bare $\mathrm{SiO}_{2}$ nanoparticles during the process of abrasion test.

To further test the tolerance to common damages, the superhydrophobic coating was then treated with tape peeling, shown in Fig. 12. It could be clearly observed that the PEW whiskers on the surface were removed by tape peeling but the nanoscale layer resisted the external force and remained. Under the combined effect of $\mathrm{SiO}_{2}$, hydroxyl acrylic resin and

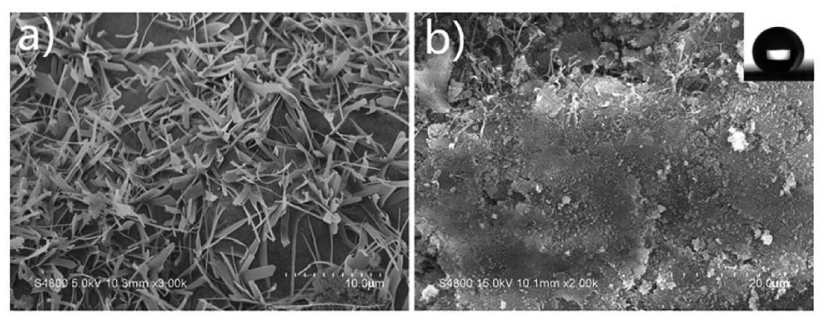

Fig. 12 SEM images of coatings before (a) and after (b) tape peeling. cross-linking agent, the coating could still maintain a favourable hydrophobicity, with a water contact angle of $140^{\circ} \pm 3^{\circ}$ on its surface.

\section{Conclusions}

A self-cleaning and mechanically durable superhydrophobic coatings with hierarchical structures were successfully constructed by a simple vapor-induced method. The immersion suspension composed of ethyl acetate, silica nanoparticles $\left(\mathrm{SiO}_{2}\right)$, hydroxyl acrylic resin, cross-linking agent and polyethylene wax (PEW). Various substrates were dip-coated in the suspension, subsequently controllably dried, forming hierarchical structure surfaces with PEW whiskers outer layer in microscale and $\mathrm{SiO}_{2}$ inner layer in nanoscale. The introduced hydroxyl acrylic resin with remarkable wear resistance and adhesion property effectively connected with $\mathrm{SiO}_{2}$ nanoparticles, ensuring the hydrophobicity and durability of inner layer. Moreover, at a suitable protocol of the dip-coating at $30^{\circ} \mathrm{C}$ and drying at $80^{\circ} \mathrm{C}$ for $2 \mathrm{~min}$ as well as $25 \mathrm{wt} \%$ of PEW, uniform PEW whiskers formed in carpet-like shapes and the coating transferred into a superhydrophobic coating with a water contact angle of $163^{\circ} \pm 5^{\circ}$, excellent self-cleaning property even under solvent-contamination. Besides, the superhydrophobic coating could bear fairly intensive knife-scratch, tape peeling and 25 cycles of sandpaper abrasion under $100 \mathrm{~g}$ of loading.

\section{Conflicts of interest}

There are no conflicts to declare.

\section{Acknowledgements}

The authors are grateful for the financial support from the National Natural Science Foundation of China (51573043), The Key Laboratory of Advanced Polymer Materials of Shanghai (Grant No. ZD 20170203) and Shanghai Leading Academic Discipline Project (B502).

\section{References}

1 H. J. Ensikat, P. Ditsche-Kuru, C. Neinhuis and W. Barthlott, Superhydrophobicity in Perfection: The Outstanding Properties of the Lotus Leaf, Beilstein J. Nanotechnol., 2011, 2, 152-161.

2 Y. Su, B. Ji, Y. Huang and K. C. Hwang, Nature's Design of Hierarchical Superhydrophobic Surfaces of a Water Strider for Low Adhesion and Low-Energy Dissipation, Langmuir, 2010, 26, 18926-18937.

3 Y. Zheng, X. Gao and L. Jiang, Directional Adhesion of Superhydrophobic Butterfly Wings, Soft Matter, 2007, 3, 178-182.

4 J. Li, L. Shi, Y. Chen, Y. Zhang, Z. Guo, B. Su and W. Liu, Stable Superhydrophobic Coatings from Thiol-Ligand Nanocrystals and their Application in Oil/Water Separation, J. Mater. Chem., 2012, 22, 9774-9781. 
5 M. Ruan, W. Li, B. Wang, B. Deng, F. Ma and Z. Yu, Preparation and Anti-Icing Behavior of Superhydrophobic Surfaces on Aluminum Alloy Substrates, Langmuir, 2013, 29, 8482-8491.

6 C. Cao, M. Ge, J. Huang, S. Li, S. Deng, S. Zhang, Z. Chen, K. Zhang, S. S. Al-Deyab and Y. Lai, Robust Fluorine-Free Superhydrophobic PDMS-ormosil@fabrics for Highly Effective Self-Cleaning and Efficient Oil-Water Separation, J. Mater. Chem. A, 2016, 4, 12179-12187.

7 S. Alexander, J. Eastoe, A. M. Lord, F. Guittard and A. R. Barron, Branched Hydrocarbon Low Surface Energy Materials for Superhydrophobic Nanoparticle Derived Surfaces, ACS Appl. Mater. Interfaces, 2016, 8, 660-666.

8 Y. Lee, S. H. Park, K. B. Kim and J. K. Lee, Fabrication of Hierarchical Structures on a Polymer Surface to Mimic Natural Superhydrophobic Surfaces, Adv. Mater., 2007, 19, 2330-2335.

9 J. Genzer and K. Efimenko, Creating Long-Lived Superhydrophobic Polymer Surfaces through Mechanically Assembled Monolayers, Science, 2000, 290, 2130-2133.

10 S. Yuan, S. O. Pehkonen, B. Liang, Y. P. Ting, K. G. Neoh and E. T. Kang, Superhydrophobic Fluoropolymer-Modified Copper Surface via Surface Graft Polymerisation for Corrosion Protection, Corros. Sci., 2011, 53, 2738-2747.

11 J. T. Han, Y. Jang, D. Y. Lee, J. H. Park, S. H. Song, D. Y. Ban and K. Cho, Fabrication of a Bionic Superhydrophobic Metal Surface by Sulfur-Induced Morphological Development, $J$. Mater. Chem., 2005, 15, 3089-3092.

12 X. Li, G. Chen, Y. Ma, L. Feng, H. Zhao, L. Jiang and F. Wang, Preparation of a Superhydrophobic Poly (Vinyl Chloride) Surface via Solvent-Nonsolvent Coating, Polymer, 2006, 47, 506-509.

$13 \mathrm{~W}$. Lee, M. K. Jin, A. Woncheol Yoo and J. K. Lee, Nanostructuring of a Polymeric Substrate with WellDefined Nanometer-Scale Topography and Tailored Surface Wettability, Langmuir, 2004, 20, 7665-7669.

14 M. Manca, A. Cannavale, L. De Marco, A. S. Arico, R. Cingolani and G. Gigli, Durable Superhydrophobic and Antireflective Surfaces by Trimethylsilanized Silica Nanoparticles-Based Sol-Gel Processing, Langmuir, 2009, 25, 6357-6362.

15 C. H. Xue, Y. R. Li, P. Zhang, J. Z. Ma and S. T. Jia, Washable and Wear-Resistant Superhydrophobic Surfaces with SelfCleaning Property by Chemical Etching of Fibers and Hydrophobization, ACS Appl. Mater. Interfaces, 2014, 6, 10153-10161.

16 X. M. Li, D. Reinhoudt and M. Crego-Calama, What do We Need for a Superhydrophobic Surface? A Review on the Recent Progress in the Preparation of Superhydrophobic Surfaces, Chem. Soc. Rev., 2007, 36, 1350-1368.

17 K. Li, X. Zeng, X. Lai and S. Chai, Study on the Anti-Abrasion Resistance of Superhydrophobic Coatings Based on Fluorine-Containing Acrylates with Different $\mathrm{Tg}$ and $\mathrm{SiO}_{2}$, RSC Adv., 2017, 7, 47738-47745.

18 J. D. Brassard, D. K. Sarkar and J. Perron, Fluorine Based Superhydrophobic Coatings, Appl. Sci., 2012, 2, 453-464.
19 U. Cengiz, M. Z. Avci, H. Y. Erbil and A. S. Sarac, Superhydrophobic Terpolymer Nanofibers Containing Perfluoroethyl Alkyl Methacrylate by Electrospinning, Appl. Surf. Sci., 2012, 258, 5815-5821.

20 S. Zhou, X. Ding and L. Wu, Fabrication of Ambient-Curable Superhydrophobic Fluoropolysiloxane/ $/ \mathrm{TiO}_{2}$ Nanocomposite Coatings with Good Mechanical Properties and Durability, Prog. Org. Coat., 2013, 76, 563-570.

21 F. Zheng, H. Deng, X. Zhao, X. Li, C. Yang, Y. Yang and A. Zhang, Fluorinated Hyperbranched Polyurethane Electrospun Nanofibrous Membrane: Fluorine-Enriching Surface and Superhydrophobic State with High Adhesion to Water, J. Colloid Interface Sci., 2014, 421, 49-55.

22 Z. Xu, Y. Zhao, H. Wang, H. Zhou, C. Qin, X. Wang and T. Lin, Fluorine-Free Superhydrophobic Coatings with $\mathrm{pH}$ Induced Wettability Transition for Controllable Oil-Water Separation, ACS Appl. Mater. Interfaces, 2016, 8, 5661-5667.

23 J. Sheng, Y. Xu, J. Yu and B. Ding, Robust Fluorine-Free Superhydrophobic Amino-Silicone $\mathrm{Oil} / \mathrm{SiO}_{2}$ Modification of Electrospun Polyacrylonitrile Membranes for WaterproofBreathable Application, ACS Appl. Mater. Interfaces, 2017, 9, 15139-15147.

24 A. Milionis, R. Ruffilli and I. Bayer, Superhydrophobic Nanocomposites from Biodegradable Thermoplastic Starch Composites (Mater-Bi), Hydrophobic Nano-silica and Lycopodium Spores, RSC Adv., 2014, 4, 34395-34404.

25 P. N. Manoudis and I. Karapanagiotis, Modification of the Wettability of Polymer Surfaces Using Nanoparticles, Prog. Org. Coat., 2014, 77, 331-338.

26 H. Ye, L. Zhu, W. Li, H. Liu and H. Chen, Constructing Fluorine-Free and Cost-Effective Superhydrophobic Surface with Normal-Alcohol-Modified Hydrophobic $\mathrm{SiO}_{2}$ Nanoparticles, ACS Appl. Mater. Interfaces, 2017, 9, 858-867. 27 S. Ramakrishna, K. S. S. Kumar, D. Mathew and C. P. R. Nair, Long-Living, Stress- and pH-Tolerant Superhydrophobic Silica Particles via Fast and Efficient Urethane Chemistry; Facile Preparation of Self-Recoverable SH Coatings, $J$. Mater. Chem. A, 2015, 3, 1465-1475.

28 M. T. Masood, J. A. Heredia-Guerrero and L. Ceseracciu, Superhydrophobic High Impact Polystyrene (HIPS) Nanocomposites with Wear Abrasion Resistance, Chem. Eng. J., 2017, 322, 10-21.

29 S. Naderizadeh, A. Athanassiou and I. S. Bayer, Interfacing Superhydrophobic Silica Nanoparticle Films with Graphene and Thermoplastic Polyurethane for Wear/ Abrasion Resistance, J. Colloid Interface Sci., 2018, 519, 285-295.

30 X. Lu, C. Zhang and Y. Han, Low-Density Polyethylene Superhydrophobic Surface by Control of Its Crystallization Behavior, Macromol. Rapid Commun., 2004, 25, 1606-1610.

$31 \mathrm{~W}$. Zhang, P. Lu, L. Qian and H. Xiao, Fabrication of Superhydrophobic Paper Surface via Wax Mixture Coating, Chem. Eng. J., 2014, 250, 431-436.

32 W. Wang, K. Lockwood, L. M. Boyd, M. D. Davidson, S. Movafaghi, H. Vahabi, S. R. Khetani and A. K. Kota, Superhydrophobic Coatings with Edible Materials, ACS Appl. Mater. Interfaces, 2016, 8, 18664-18668. 
33 X. Wang, Z. Li, M. Zhang, T. Fan and B. Cheng, Preparation of a Polyphenylene Sulfide Membrane from a Ternary Polymer/Solvent/Non-solvent System by Thermally Induced Phase Separation, RSC Adv., 2017, 7, 10503-10516.

34 A. Milionis, K. Dang and M. Prato, Liquid Repellent Nanocomposites Obtained from One-step Water-based Spray, J. Mater. Chem. A, 2015, 3, 12880-12889.

35 D. Aslanidou, I. Karapanagiotis and C. Panayiotou, Tuning the Wetting Properties of Siloxane-Nanoparticle Coatings to Induce Superhydrophobicity and Superoleophobicity for Stone Protection, Mater. Des., 2016, 108, 736-744.

36 D. Aslanidou, I. Karapanagiotis and C. Panayiotou, Superhydrophobic, Superoleophobic Coatings for the Protection of Silk Textiles, Prog. Org. Coat., 2016, 97, 44-52. 37 Y. Lu, S. Sathasivam, J. Song, C. R. Crick, C. J. Carmalt and I. P. Parkin, Robust Self-Cleaning Surfaces that Function When Exposed to Either Air or Oil, Science, 2015, 347, 1132-1135. 